\title{
A PDMS-Coated Optical Fiber Bragg Grating Sensor for Enhancing Temperature Sensitivity
}

\author{
Chang-sub Park \\ Department of Sensor and Display Engineering, Kyungpook National University, \\ Sankyuk-dong, Daegu 702-701, Korea \\ Kyung-Il Joo \\ School of Electrical Engineering and Computer Science, Kyungpook National University, \\ Sankyuk-dong, Daegu 702-701, Korea \\ Shin-Won Kang and Hak-Rin Kim* \\ School of Electronics Engineering, Kyungpook National University, \\ Sankyuk-dong, Daegu 702-701, Korea
}

(Received September 20, 2011 : revised October 12, 2011 : accepted October 17, 2011)

\begin{abstract}
We proposed a poly-dimethylsiloxane (PDMS)-coated fiber Bragg grating (FBG) temperature sensor for enhancing temperature sensitivity. By embedding the bare FBG in a temperature-sensitive elastomeric polymer, the temperature sensitivity of the proposed structure could be effectively improved by 4.2 times higher than those of the conventional bare-type FBG sensors due to the high thermal expansion coefficient of the PDMS. We analyzed the temperature-sensitivity enhancement effect with the increased Bragg wavelength shift in our structure and dependence on the temperature sensitivity with respect to the cross-section area of the PDMS.
\end{abstract}

Keywords: Temperature sensor, Optical fiber sensor, Fiber Bragg grating, Elastomeric polymer, Thermal expansion

OCIS codes : (060.3735) Fiber Bragg grating; (160.5470) Polymers; (230.3990) Micro-optical devices;

(280.4788) Optical sensing and sensors

\section{INTRODUCTION}

In the past decades, fiber Bragg grating (FBG) sensors have been studied extensively for sensing several types of physical parameters such as temperature, strain, bending, and pressure [1-6] with optical methods. FBG sensors have the advantages of immunity to electromagnetic interference, compact structure, simple fabrication, and easy construction of long range monitoring system by using wavelengthmultiplexing schemes with cascaded FBGs. However, for temperature sensing itself, the temperature sensitivities of bare FBG sensors are low due to the low thermal expansion coefficient of silica with temperature variation [1]. To increase the temperature sensitivity of the FBG sensor, several types of modified FBG sensors have been proposed. The earlier researchers studied deposition methods using metal or metal complex compounds with high thermal expansion coefficients to enhance the temperature sensitivity [7-10]. However, the metal deposition process on the silica fiber requires complex fabrication steps, for which it is difficult to control the deposition layer precisely due to the radial shape of optical fibers. The residual stress, produced during the metal deposition process, on the interface between the deposited metal and the glass also makes it difficult to design the resonant peaks of the metal-deposited FBG sensors precisely [10]. For the case of using polymers for enhancing the temperature sensitivity of FBG sensors, the fabrication process can be comparatively simple because the polymer cladding

\footnotetext{
*Corresponding author: rineey@ee.knu.ac.kr

Color versions of one or more of the figures in this paper are available online.
} 
layer can be made simply by a general coating process, but their temperature sensitivities are not good due to the low thermal expansion coefficient $[11,12]$. In addition, the thermal dimensional stability of a polymer should be considered to be used for the temperature-sensitivity-enhancing materials because the thermally expanded volume of most polymers cannot be restored completely to an initial volume after cooling.

In this study, we propose a poly-dimethylsiloxane (PDMS)coated FBG temperature sensor, where the PDMS is used for enhancing temperature sensitivity as shown in Fig. 1. The thermal expansion coefficient of the PDMS is much higher than those of metal materials previously used for enhancing temperature sensitivity in metal-coated FBG sensors as shown in Table $1[13,14]$. Thus, the temperature sensitivity can be effectively enhanced by the tensile force induced by the thermally expanded PDMS. Because the PDMS is a chemically stable elastomeric polymer, the thermally shifted resonant peak in our structure moves back to an initial wavelength without dimensional stability problems. In addition, we can make the PDMS jacket on the FBG very easily due to its good adhesion on the silica surface by using a simple molding process [15]. However, the enhancement of the temperature sensitivity in the PDMScoated FBG sensor is not much higher than the large thermal expansion coefficient of the PDMS itself. It is because the Young's modulus of the silica is much higher than that of the elastomeric PDMS. Here, we give an analytic model to describe the enhanced Bragg wavelength shift of the proposed structure in response to the increased temperature, and we demonstrate the temperature sensitivity according to crosssection areas of the PDMS jackets.

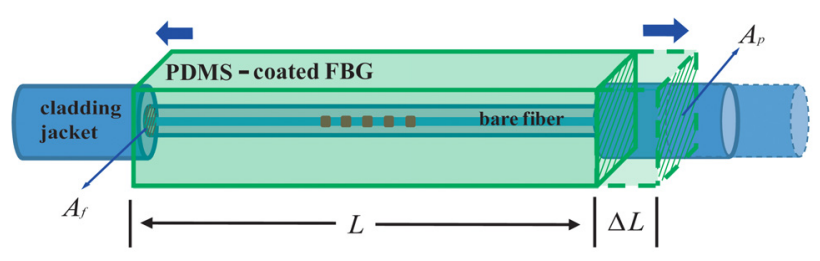

FIG. 1. The schematic illustration of the PDMS-coated FBG sensor designed for enhancing temperature sensitivity by using thermal expansion effect of the PDMS jacket.

TABLE 1. The thermal expansion coefficients of materials used for enhancing temperature sensitivity of optical FBG sensors.

\begin{tabular}{c|cc}
\hline \hline Materials & \multicolumn{2}{|c}{$\begin{array}{c}\text { Thermal expansion coefficient } \\
\left(\mathrm{m} / \mathrm{m}^{\circ} \mathrm{C}\right)\end{array}$} \\
\hline $\mathrm{Al}$ & $23.03 \times 10^{-6}$ & ${ }^{[13]}$ \\
\hline $\mathrm{Ti}$ & $8.35 \times 10^{-6}$ & {$[13]$} \\
\hline $\mathrm{Ni}$ & $13.3 \times 10^{-6}$ & {$[13]$} \\
\hline $\mathrm{PDMS}$ & $300 \times 10^{-6}$ & {$[14]$} \\
\hline
\end{tabular}

\section{THEORY}

The FBG structure can measure the temperature variation by monitoring the shift $\left(\Delta \lambda_{B}\right)$ in the Bragg resonance wavelength $\left(\lambda_{B}\right)$ induced by surrounding temperature variation. When temperature is varied, $\lambda_{B}$ is shifted to longer or shorter wavelength due to the thermally varied effective refractive index of the fiber and the thermally varied Bragg grating periodicity. In our PDMS-coated FBG temperature sensor, the $\lambda_{B}$ is additionally shifted by the tensile force of the thermally expanded PDMS jacket. Considering the reciprocal force $\left(F_{r}\right)$ equally applied to the optical fiber and the PDMS jacket in our PDMS-coated FBG sensor, the tensile forces onto the fiber $\left(F_{t f}\right)$ and onto the PDMS jacket $\left(F_{t p}\right)$ can be expressed as follows.

$$
F_{t f}=F_{f}+F_{r}, \quad F_{t p}=F_{p}-F_{r}
$$

where $F_{f}$ and $F_{p}$ are the thermally induced tensile forces applied onto the fiber and PDMS jacket, respectively, when it is assumed that there is no force interaction between the optical fiber and the PDMS jacket at the interface. Thus, both $F_{f}$ and $F_{p}$ can be obtained with the material parameters of the thermal expansion coefficients. Since the thermally induced length variation $(\Delta L)$ is the same at the fiber and the PDMS jacket in our PDMS-coated FBG sensor, $\Delta L$ can be expressed by modification of Eq. (1) as follows.

$$
\Delta L=\frac{F_{f}+F_{r}}{k_{f}}=\Delta L_{f}+\Delta L_{f}^{r}=\frac{F_{p}-F_{r}}{k_{p}}=\Delta L_{p}-\Delta L_{p}^{r}
$$

where $k_{f}$ and $k_{p}$ are the elastic constants of the bare fiber and the PDMS, respectively. $\Delta L_{f}$ and $\Delta L_{p}$ are the thermally induced length variation of the fiber and the PDMS, respectively, assuming that there is no interaction force between the fiber and the PDMS. $\Delta L_{f}^{r}$ and $\Delta L_{p}^{r}$ are the additional length variation of the fiber and the PDMS, respectively, induced by the reciprocal force in the PDMS-coated FBG structure. Considering that the thermal expansion coefficient of the PDMS is much higher than that of the silica fiber, we can see that the thermal expansion of the PDMS is restricted by the amount of $\Delta L_{p}^{r}$, whereas the thermal expansion of the FBG is enhanced by $\Delta L_{f}^{r}$. Since $F_{r}$ can be written from Eqs. (1) and (2) by

$$
F_{r}=k_{f} \Delta L_{f}^{r}=k_{p} \Delta L_{p}^{r}
$$

where $\Delta L$ can be expressed after some calculations by using the Young's modulus equation [16] as follows.

$$
\Delta L=\Delta L_{f}+\left(\Delta L_{p}-\Delta L_{f}\right) \cdot \frac{A_{p} E_{p}}{A_{p} E_{p}+A_{f} E_{f}}
$$

Here, $A_{p}$ and $A_{f}$ denote the cross-section area of the PDMS 
and the optical fiber, respectively, in our PDMS-coated FBG structure. $E_{p}$ and $E_{f}$ are the Young's modulus values of the PDMS and the silica fiber, respectively. Equation (4) shows that the thermally induced length variation in our PDMScoated FBG structure can be expressed by the device dimension parameters and the material parameters of the thermal expansion coefficients.

Considering the thermally induced refractive index variation in the silica fiber, the Bragg wavelength shift of the PDMS-coated FBG sensor according to temperature change can be written as follows.

$$
\Delta \lambda_{B}=\lambda_{B}\left(\alpha_{n}+\alpha_{f}+\alpha_{p}\right) \Delta T
$$

where $\alpha_{n}, \alpha_{f}, \alpha_{p}$, and $\Delta T$ are the thermo-optic coefficient $\left(\sim 8.6 \times 10^{-6} \mathrm{C}\right)$ of the silica fiber, the thermal expansion coefficient $\left(\sim 0.55 \times 10^{-6} \mathrm{~m} / \mathrm{m}^{\circ} \mathrm{C}\right)$ of the fiber, the thermal expansion coefficient $\left(\sim 300 \times 10^{-6} \mathrm{~m} / \mathrm{m}^{\circ} \mathrm{C}\right)$ of the PDMS, and the temperature variation amount, respectively $[13,17]$. Since $\Delta L_{f}$ and $\Delta L_{p}$ in Eq. (4) are written by $\Delta L_{f}=L \Delta T \alpha_{f}$ and $\Delta L_{p}=L \Delta T \alpha_{p}$, respectively, $\Delta \lambda_{B}$ is finally derived from Eqs. (4) and (5) as follows,

$$
\Delta \lambda_{B}=\lambda_{B} \alpha_{n} \Delta T+\lambda_{B}\left(1-\hat{P}_{e}\right) \frac{\Delta L}{L}=\lambda_{B}\left[\alpha_{n}+\left(1-\hat{P}_{e}\right) \cdot\left\{\alpha_{f}+\left(\alpha_{p}-\alpha_{f}\right)\right\} \cdot \frac{A_{p} E_{p}}{A_{p} E_{p}+A_{f} E_{f}}\right] \cdot \Delta T,
$$

where $\hat{P}_{e}$ is the effective photoelastic coefficient $(\sim 0.212)$ of the fiber [17].

\section{EXPERIMENTAL RESULTS}

\subsection{Fabrication and Measurement of the PDMS-coated FBG Temperature Sensor}

For the PDMS-coated FBG sensor, the FBG (SMF-28) with total longitudinal grating length of $10 \mathrm{~mm}$ was utilized in our experiments, which presented the central wavelength of $1549.6 \mathrm{~nm}$, the $3 \mathrm{~dB}$ bandwidths of about $0.1 \mathrm{~nm}$ and $0.2 \mathrm{~nm}$, and the peak reflectivity of about $90 \%$. Before coating the PDMS onto the FBG, the cladding surface of the FBG was pretreated with the oxygen plasma at $30 \mathrm{~W}$ for 30 seconds to enhance the interfacial adhesion between the PDMS and the optical bare fiber [18]. The PDMS jacket was fabricated with a molding process by using the PDMS with a 10:1 mixing ratio of precursor of elastic material (Sylgard 184-A) and hardener (Sylgard 184-B), where the length of the PDMS jacket was $L=70 \mathrm{~mm}$. The FBG was precisely aligned to be positioned at the center of the PDMS block. In the mold, the prepolymer of the mixtures was polymerized by baking at $60^{\circ} \mathrm{C}$ for 12 hours. Finally, the PDMS-coated FBG sensors were completed by removing the mold. Three kinds of the PDMS-coated FBG sensors with the different cross-section areas $\left(A_{p}=5 \times 5 \mathrm{~mm}^{2}\right.$, $A_{p}=10 \times 10 \mathrm{~mm}^{2}, A_{p}=20 \times 20 \mathrm{~mm}^{2}$ ) of the PDMS jackets were prepared to check the temperature sensitivity depending

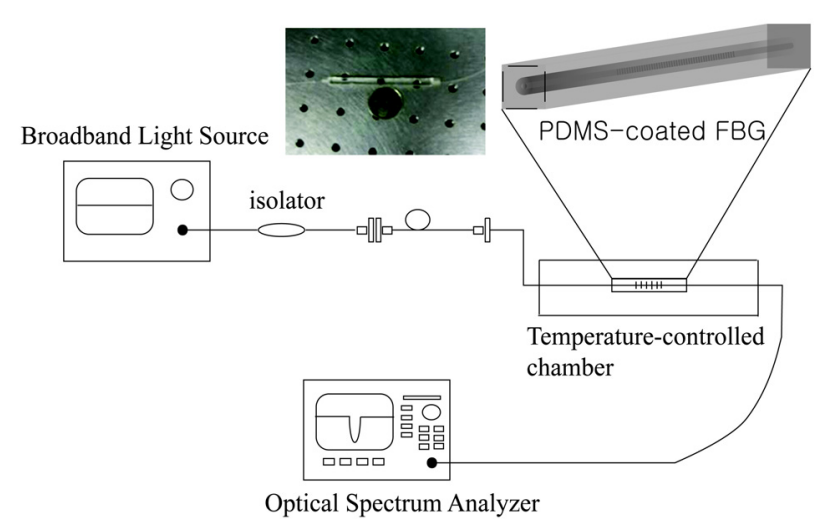

FIG. 2. Experimental setup for measuring temperature sensitivity of the PDMS-coated FBG sensor.

on the device dimension condition of the PDMS jacket and to compare the experimental results with the derived analytic model. For the PDMS-coated FBG sensor with the PDMS jacket with $A_{p}=5 \times 5 \mathrm{~mm}^{2}$, the bare FBG with the 3 $\mathrm{dB}$ bandwidth of $0.1 \mathrm{~nm}$ was used. For the others, the 3 $\mathrm{dB}$ bandwidth of the bare FBG was $0.2 \mathrm{~nm}$.

As shown in Fig. 2, a broadband light source (Opto-Link Corporation Ltd. OLS15CLGB-15FA) was connected to the proposed FBG sensor with an optical isolator. The transmission spectra of the PDMS-coated FBG sensor were monitored using an optical spectrum analyzer (OSA, Ando AQ-6315A) with a resolution bandwidth of $0.05 \mathrm{~nm}$. During the experiments, temperature was precisely controlled from $30^{\circ} \mathrm{C}$ to $120^{\circ} \mathrm{C}$ by using a temperature-controlled pyrostat.

\subsection{Enhancement of Temperature Sensitivity by the PDMS Jacket}

Figure 3 shows the transmission spectra of the PDMScoated FBG sensors at various temperatures, where the crosssection areas of the PDMS-jackets are $A_{p}=5 \times 5 \mathrm{~mm}^{2}, A_{p}=$ $10 \times 10 \mathrm{~mm}^{2}$, and $A_{p}=20 \times 20 \mathrm{~mm}^{2}$. In all cases, the measured $\Delta \lambda_{B}$ 's increase with increasing temperature due to the thermal expansion effect of the PDMS jackets as we can see from Eq. (6). Figure 3 shows that the shapes as well as the 3 $\mathrm{dB}$ bandwidths of the thermally shifted transmission spectra are the same during the temperature sweep which means that the tensile force induced by the PDMS jacket is uniform without producing additional bending distortions. In addition, the thermally shifted Bragg wavelengths are moved back to the initial $\lambda_{B}$ after cooling to initial temperature irrespective of the tensile stress amount induced by the thermally expanded PDMS jacket within our experiment conditions, which shows that the adhesion between the silica cladding surface and the PDMS jacket is good and, due to its good elasticity, there is no dimensional stability problem or residual stress problem in our PDMS jacket

Figure 4(a) shows $\Delta \lambda_{B}$ 's of the PDMS-coated FBG sensors for various cross-section areas of the PDMS jacket according to temperature variation, where the dots and the lines 


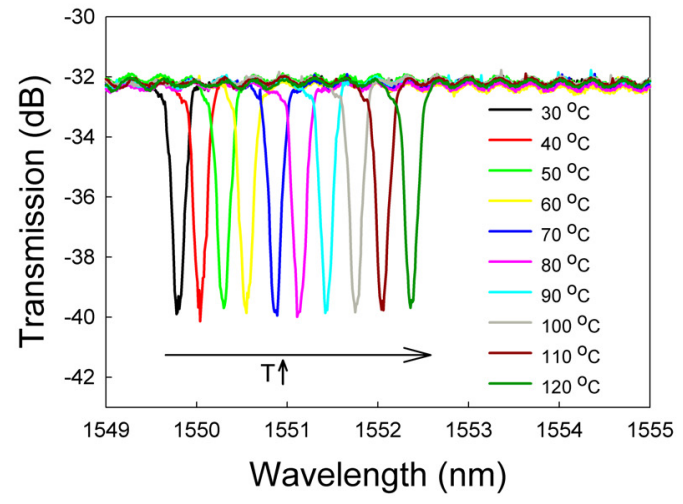

(a)

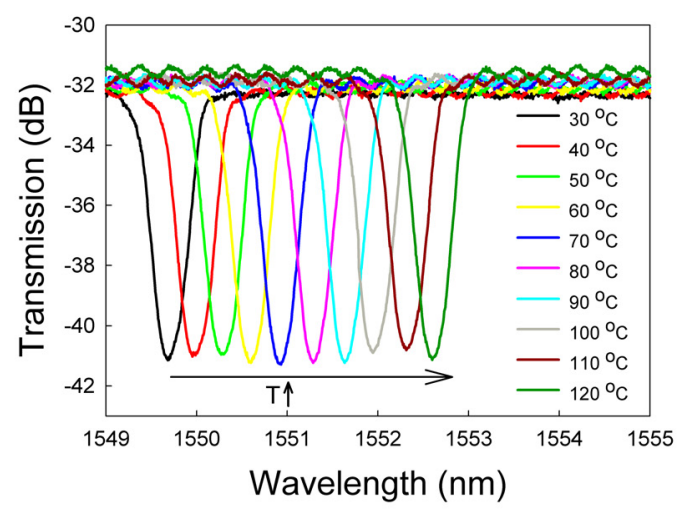

(b)

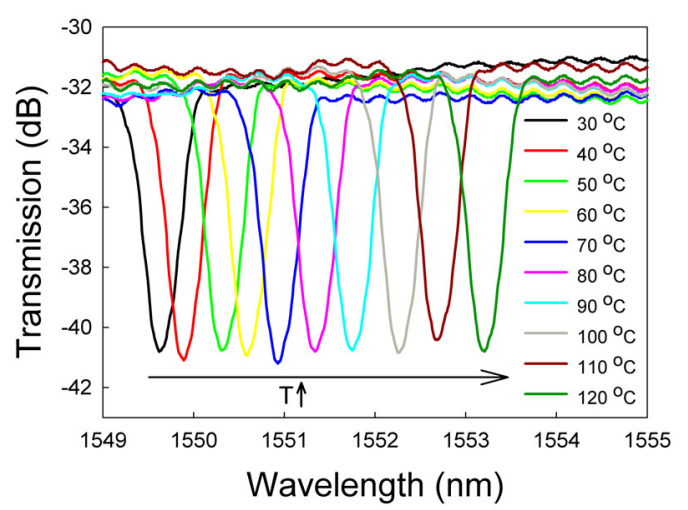

(c)

FIG. 3. The transmission spectra of the PDMS-coated FBG sensors at various temperatures, where the cross-section areas of the PDMS-jackets are (a) $A_{p}=25 \mathrm{~mm}^{2}$, (b) $A_{p}=100 \mathrm{~mm}^{2}$, and (c) $A_{p}=400 \mathrm{~mm}^{2}$.

denote the experimental results and the simulation results from Eq. (6). For comparison, $\Delta \lambda_{B}$ 's of the bare FBG without the PDMS jacket are plotted together. In all cases, $\Delta \lambda_{B}$ increases linearly with increasing $\Delta T$ as expected from Eq. (6). However, the temperature sensitivity of the bare FBG was $0.010 \mathrm{~nm} /{ }^{\circ} \mathrm{C}$. On the other hand, the temperature sensitivities of the PDMS-coated FBG sensors were much higher than that of the bare FBG. They were enhanced to 0.026 $\mathrm{nm} /{ }^{\circ} \mathrm{C}$ (for $A_{p}=25 \mathrm{~mm}^{2}$ ), $0.033 \mathrm{~nm} /{ }^{\circ} \mathrm{C}$ (for $A_{p}=100 \mathrm{~mm}^{2}$ ),

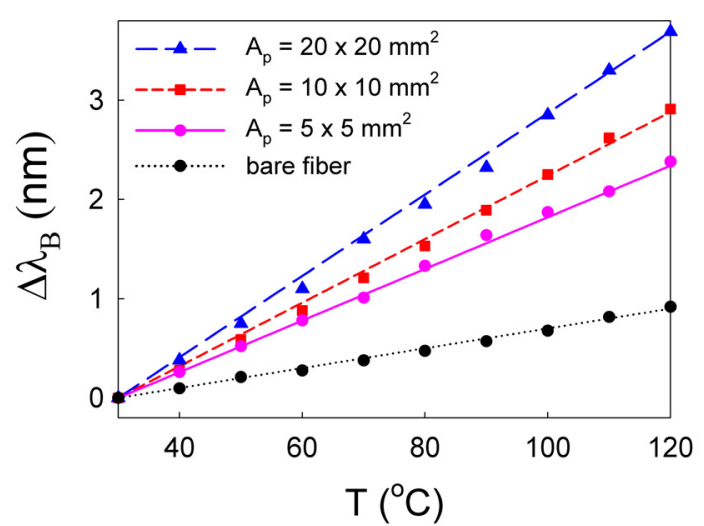

(a)

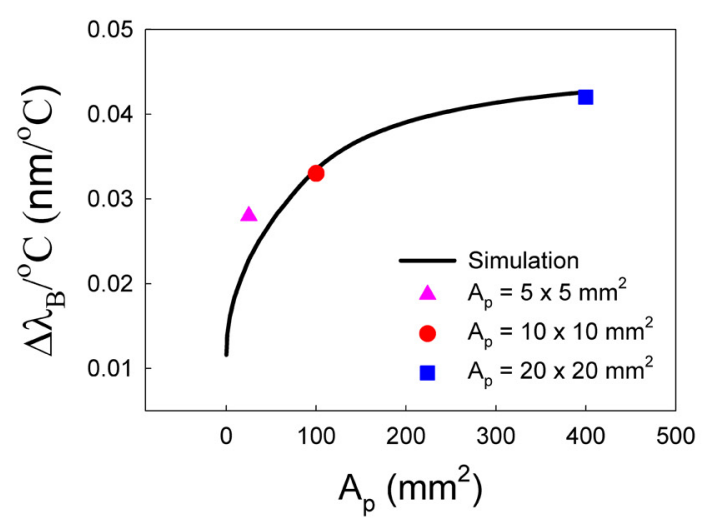

(b)

FIG. 4. (a) $\Delta \lambda_{B}$ 's of the PDMS-coated FBG sensors as a function of temperature for various cross-section areas of the PDMS. (b) The temperature sensitivities of the PDMS-coated FBG sensors for various cross-section areas of the PDMS jackets. The dots and the lines denote the experimental results and the simulation results from Eq. (6), respectively.

and $0.042 \mathrm{~nm} /{ }^{\circ} \mathrm{C}$ (for $A_{p}=400 \mathrm{~mm}^{2}$ ). Equations (4) and (6) show that the tensile force thermally induced by the PDMS jacket can be effectively delivered to the inner FBG with increasing $A_{p}$. Thus, the temperature sensitivity increases with increasing $A_{p}$ of the PDMS-coated FBG sensor. The temperature sensitivity of the PDMS-coated FBG sensor is comparable to that $\left(\sim 0.048 \mathrm{~nm} /{ }^{\circ} \mathrm{C}\right)$ of the metal-coated FBG temperature sensor [10]. However, the PDMS-coated FBG sensor can be made by the simple molding process without any residual stress problem during fabrication compared to conventional metal-coated FBG sensors so that the initial resonance wavelength as well as the amount of the temperatureinduced resonant wavelength shift can be precisely designed.

The material parameters used for theoretic values, as depicted in Fig. 4, from Eq. (6) are that the diameter of the bare fiber is $125 \mu \mathrm{m}$, and the Young's moduli of the PDMS and the bare fiber are $E_{p}=750 \mathrm{Kpa}$ and $E_{f}=72 \mathrm{Gpa}$, respectively $[19,20]$. For the thermal expansion coefficient of the PDMS, the fitted value $\left(\alpha_{p}=312 \times 10^{-6} \mathrm{~m} / \mathrm{m}^{\circ}{ }^{\circ} \mathrm{C}\right)$ from the experimental results was used. It was slightly higher 
than that from the data sheet shown in Table 1. The thermal expansion coefficient of PDMS varies slightly depending on the mixing ratio of the prepolymers and the irregularly entangled polymer chain network structure [21]. For the PDMS-coated FBG sensors with the PDMS jacket conditions of $A_{p}=100 \mathrm{~mm}^{2}$ and $A_{p}=400 \mathrm{~mm}^{2}$, the simulation results of $\Delta \lambda_{B}$ with Eq. (6) and the same material conditions matched well with the experimental results. However, for the case of $A_{p}=25 \mathrm{~mm}^{2}$, the analytic results agreed with the experimental results when $A_{p}=49 \mathrm{~mm}^{2}$ was used for the crosssection area of the PDMS-jacket at the same material conditions. The difference might originate in our molding process.

Figure 4(b) shows the temperature sensitivity of the PDMScoated FBG sensor for various values of $A_{p}$, which was plotted with Eq. (6). For comparison, the experimental values obtained with Fig. 4(a) were plotted together. Figure 4(b) shows that the temperature sensitivity enhancement effect by the PDMS jacket is high with increasing $A_{p}$ at small values of $A_{p}$. However, the enhancement effect becomes saturated at higher values of $A_{p}$. From Eq. (6), we can see that the enhancement of $\Delta \lambda_{B} / \Delta T$ by increasing $A_{p}$ is saturated when $A_{p} \cdot E_{p}$ becomes larger than $A_{f} \cdot E_{f}$. Our results and the analytic solution show that the temperature sensitivity of the FBG can be effectively enhanced only with thin layer of the PDMS jacket. In addition, we can use $A_{p}$ as a device design parameter to control the temperature sensitivity of the FBG sensor. It is also expected that the chirp-shaped spectra under temperature variation can be easily tailored by using the PDMS jacket with $A_{p}$ variation along the fiber axis direction. Previously, we have shown that the selective chemical sensing is available with the PDMS-coated FBG structure by using the selective swelling effect of the PDMS under chemical exposure [15]. The previous analytic model, derived for the selective chemical sensing, can be improved with this work.

\section{CONCLUSION}

We proposed a highly temperature-sensitive FBG structure, where the bare FBG was coated with the elastomeric PDMS jacket layer. The analytic model was also derived, which matched well with the experimental results. The experimental results as well as the theoretical model showed that the amount of the tensile force onto the PDMS-coated FBG, induced by temperature variation, was highly dependent on the cross-section area of the PDMS jacket, which means that we can use our structure for enhancing temperature sensitivity effectively or for tailoring temperature-dependent spectral shape change for several other sensing applications with modified PDMS shapes. The proposed PDMS-coated FBG sensor is expected to be widely used for various applications due to its high temperature sensitivity, easy fabrication, high durability and stability without interfacial residual stress between the PDMS and the fiber.

\section{ACKNOWLEDGMENT}

This work was supported by Basic Science Research Program through the National Research Foundation of Korea (NRF) grant funded by the Korea government (MEST) (20110001083) and results of a study on the "Human Resource Development Center for Economic Region Leading Industry" Project, supported by the Ministry of Education, Science \& Technology (MEST) and the National Research Foundation of Korea (NRF).

\section{REFERENCES}

1. A. Othonos and K. Kalli, Fiber Bragg Gratings Fundamentals and Applications in Telecommunications and Sensing (Artech House, Boston, USA, 1999).

2. Y. J. Rao, "Recent progress in applications of in-fiber Bragg grating sensors," Opt. Laser Eng. 31, 297-324 (1999).

3. M.-H. Song, "A wideband interferometric wavelength shift demodulator of fiber Bragg grating strain sensor," J. Opt. Soc. Korea 3, 64-68 (1999).

4. S.-M. Lee and J.-S. Sirkis, "Hydrogen sensor based on palladiumattached fiber Bragg grating," J. Opt. Soc. Korea 3, 69-73 (1999).

5. X. Shu, K. Chisholm, I. Felmeri, K. Sugden, A. Gillooly, L. Zhang, and I. Bennion, "Highly sensitive transverse load sensing with reversible sampled fiber Bragg gratings," Appl. Phys. Lett. 83, 3003-3005 (2003).

6. G. Chen, L. Liu, J. Jia, J. Yu, L. Xu, and W. Wang, "Simultaneous strain and temperature measurements with fiber Bragg grating written in novel Hi-Bi optical fiber," IEEE Photon. Technol. Lett. 16, 221-223 (2004).

7. S. Gupta, T. Mizunami, T. Yamao, and T. Shimomura, "Fiber Bragg grating cryogenic temperature sensors," Appl. Opt. 35, 5202-5205 (1996).

8. C. Lupi, F. Felli, L. Ippoliti, M. A. Caponero, M. Ciotti, V. Nardelli, and A. Paolozzi, "Metal coating for enhancing the sensitivity of fibre Bragg grating sensors at cryogenic temperature," Smart Mater. Struct. 14, N71-N76 (2005).

9. J. Jung, H. Nam B. Lee, J. O. Byun, and N. S. Kim, "Fiber Bragg grating temperature sensor with controllable sensitivity," Appl. Opt. 38, 2752-2754 (1999).

10. G.-C. Lin, L. Wang, C. C. Yang, M. C. Shin, and T. J. Chuang, "Thermal performance of metal-clad fiber Bragg grating sensor," IEEE Photon. Technol. Lett. 10, $406-408$ (1998).

11. J. Long, W. Zhang, H. Zhang, B. Liu, J. Zhao, Q. Tu, G. Kai, and X. Dong, "An embedded FBG sensor for simultaneous measurement of stress and temperature," IEEE Photon. Technol. Lett. 18, 154-156 (2006).

12. P. Lu, L. Men, and Q. Chen, "Resolving cross sensitivity of fiber Bragg gratings with different polymeric coatings," Appl. Phys. Lett. 92, 171112 (2008).

13. D. R. Lide, CRC Handbook of Physics and Chemistry (CRC Press, Boca Raton, FL, USA, 2003).

14. Dow Corning Data Sheet, "www2.dowcorning.com/DataFiles /090007c88020b9a3.pdf".

15. C.-s. Park, Y. Han, K.-I. Joo, Y. W. Lee, S.-W. Kang, and H.-R. Kim, "Optical detection of volatile organic compounds 
using selective tensile effects of a polymer-coated fiber Bragg grating," Opt. Express 18, 24753-24761 (2010).

16. D. H. Young, R. A. Freedman, T. R. Sandin, and A. L. Ford, University Physics (Addison-Wesley, San Francisco, USA, 1992).

17. G. Meltz and W. W. Morey, "Bragg grating formation and germanosilicate fiber photosensitivity," Proc. SPIE 1516, 185-199 (1991).

18. S. Bhattacharya, A. Datta, J. M. Berg, and S. Gangopahyay, "Studies on surface wettability of Poly(Dimethyl)Siloxane (PDMS) and glass under Oxygen-plasma treatment and correlation with bond strength,” J. Microelectromech. Syst. 14,
590-597 (2005).

19. F. P. Mallinder and B. A. Proctor, "Elastic constants of fused silica as a function of large tensile strain," Phys. Chem. Glasses 2, 91-103 (1964).

20. D. Armani, C. Liu, and N. Aluru, "Re-configurable fluid circuits by PDMS elastomer micromachining," in Proc. IEEE Micro Electro Mechanical Systems (Orlando, FL, USA, 1999), pp. 91-103.

21. J. Mark, K. Ngai, W. Graessley, L. Mandelkern, E. Samulski, J. Koenig, and G. Wignall, Physical Properties of Polymers (Cambridge University Press, Cambridge, UK, 2004). 